\title{
Brasilien auf der Suche nach einer modernen Verfassung: die Verfassung der Republik der Vereinigten Staaten von Brasilien als kopiertes Original ${ }^{1}$
}

\section{Herkunft und Verbreitung des modernen Konstitutionalismus in Lateinamerika}

Wenn heute von »Verfassung « die Rede ist, ist stets die förmliche, die geschriebene, die juridifizierte Verfassung eines Staates gemeint, also das Staatsverfassungsgesetz oder - so der lateinamerikanische Sprachgebrauch - die »Constitución Política «. Die Verfassung in diesem urkundlichen Sinne ist die prominenteste staatspolitische Erfindung der Moderne. Als Besiegelungsform erfolgreicher Revolutionen entstanden, gilt sie heute als die Organisationsform souveräner Staatlichkeit par excellence. Nicht zufällig hat sie Weltkarriere gemacht: kaum ein Staat heute, der ohne förmliche Verfassung wäre.

Historisch fällt das Erscheinen der ersten modernen Verfassung in die Zeit der »Glorious Revolution« in England (1688). Das Original wird in Cromwell's »Instrument of Government « von 1653 gesehen, insofern dieser Verfassungsakt bereits inmitten der Blütezeit des Absolutismus den Sturz der Monarchie und die Machtergreifung des Parlaments beurkundete (Grimm,1991:105). Die eigentliche Bedeutungsgeschichte der geschriebenen Verfassung aber beginnt erst einhundert Jahre später, im letzten Drittel des 18. Jahrhunderts, als Form der Festschreibung der Errungenschaften der liberalen Revolutionen und der Errichtung des republikanischen Staats. Die beiden großen Verfassungswerke, die »Constitution of the United States of America « (1787) und die »Constitution Française $(1791 ; 1793)$, wirkten sofort beispielhaft und fanden schon wenige Jahrzehnte später Nachahmer nicht nur in Europa, sondern auch in anderen Erdteilen, besonders in Südamerika. Die aus den Unabhängigkeitskämpfen seit 1810 hervorgegangenen Staaten des Subkontinents gründeten sich ausnahmslos als Verfassungsstaaten und legten in ihren »cartas políticas « die Strukturen der nationalen Staatsorganisation fest sowie, vor allem, die Regeln und Verfahren für den Erwerb und die Ausübung der Regierungsgewalt. Der globale Siegeszug des Konstitutionalismus war unaufhaltsam. Obzwar im ganzen 19. Jahrhunderts noch von der Reaktion bekämpft, hatte sich im 20. Jahrhundert die Verfassung als rechtliche Urkundsform der Errichtung und Behauptung nationaler Staatlichkeit, Selbstbestimmung und Unabhängigkeit weltweit durchgesetzt.

Innerhalb dieser globalen Entwicklung fällt der lateinamerikanische Konstitutionalismus durch besonderen Eifer auf. Ausweislich der historischen Zählstatistik eines internationalen Verfassungsregisters (Dippel,1998) halten im weltweiten Vergleich die lateinamerikanischen Staaten den Weltrekord im Erlass von Verfassungen. Beispielsweise gab sich die Dominikanische Republik bis 1952, also innerhalb von 120 bis 130 Jahren, bereits ganze 25 Verfassungen, Haiti 22 Verfassungen und Bolivien 15

1 Vortrag auf dem Symposium «Brasilien im amerikanischen Kontext” am Ibero-Amerikanischen Institut zu Berlin (Oktober 2002). 
Verfassungen. Seitdem ist auf dem Subkontinent der Umsatz von Verfassungen noch erheblich gesteigert worden. In den 80er bis 90er Jahren hat die sog. Demokratisierungswelle nahezu allen Völkern Lateinamerikas neue Verfassungen beschert (Chile 1980/1992; Brasilien 1988; Kolumbien 1991; Perú 1993; Argentinien 1994; México 1996; Venezuela 1999; Cuba 1976/2002). In dieser Statistik bildet Brasilien mit nicht mehr als acht Bundesverfassungen im langen Zeitraum von 164 Jahren nur scheinbar eine Ausnahme. Zählt man nämlich die Länderverfassungen (»Constituições Estaduais«) hinzu sowie die Verfassungsdiktate von Regierungen (»Atos Institucionais«) und die Verfassungsnovellen (»Emendas Constitucionais«), erhöht sich die Gesamtzahl der brasilianischen Verfassungsurkunden schnell auf über 200 Formalakte. Allein die geltende Verfassung von 1988 ist in den 14 Jahren ihres Bestehens 38 Mal geändert worden (Stand Juni 2002). Bedenkt man weiterhin die äußerst aufwendigen Verfahren der Verfassungsgebung mit ihren unzähligen parlamentarischen Textentwürfen, Zwischen- und Endfassungen (Paul,1994:200), dann darf Brasilien als ein wahrer Hort des lateinamerikanischen Verfassungsenthusiasmus gelten.

Die Universalgeschichte des modernen, republikanisch demokratischen Konstitutionalismus verzeichnet nicht nur den weltweiten Gebrauch der urkundlichen Verfassungsform, sondern auch den globalen Transfer inhaltlicher Standards. Auffällig ist, dass trotz aller Vielfalt der nationalstaatlichen Ausgestaltungen alle modernen Grundgesetze und Verfassungen in ihren materiellen Gehalten und funktionalen Einrichtungen mit dem originären Modell übereinstimmen, das die ersten Verfassungsgeber gegen Ende des 18. Jahrhunderts in den USA und Frankreich geschaffen haben (Löwenstein,1975:141). Alle modernen Verfassungen bekennen sich zum Prinzip der Volkssouveränität als der Quelle der Legitimität öffentlicher Gewalt, alle institutionalisieren das Prinzip der Gewaltenteilung und weisen die Staatsfunktionen getrennten Machtträgern zu, alle sehen Wahlen für Volksvertretungen und Regierungen vor, alle deklarieren sich als Wertordnungen in Gestalt von Bekenntnissen zu Menschenrechten oder religiösen Ethiken und fügen Grundrechtskataloge an. Alle modernen Verfassungen verfolgen explizit oder implizit den Zweck, die Allmacht des Leviathan zu beschränken, die Ausübung staatlicher Gewalt zu reglementieren und den Bürgern individuelle Freiheiten zu gewährleisten. In diesem weitläufigen Sinne sind alle modernen Verfassungen der Welt Abkömmlinge des demokratischen Konstitutionalismus, der mit den Verfassungen der sich als souveräne Staaten proklamierenden amerikanischen Kolonien (»colonial charters «) und endgültig mit der Gründungsverfassung der USA von 1787 rechtsgeschichtliche Urständ gefeiert hat. Zweifellos gehören auch die Verfassungen Lateinamerikas diesem geschichtlichen Kontext an. Auch sie sind vom Ideengut der Großen Liberalen Revolution geprägt und haben sich formal wie inhaltlich am vorbildlichen angloamerikanischen Modell ausgerichtet.

Damit drängt sich die Frage auf, die seither die patriotischen Gemüter Lateinamerikas beschäftigt und bis heute nicht zur Ruhe kommen lässt, die Frage nämlich, ob denn die lateinamerikanischen Verfassungen nichts als bloße Derivate des US-amerikanischen Originals sind, also nichts als bloße Importe aus dem weltweiten Transfer verfassungsjuristischer Ideen, Institutionen und Formen, der im 19. Jahrhundert in Blüte war. Oder ob nicht vielmehr in Lateinamerika, unter den Bedingungen der Grün- 
derstaaten und dem Einfluss ihrer caudillistischen Traditionen ein Verfassungstypus sui generis entstanden ist und mit ihm das Paradigma eines genuin lateinamerikanischen Konstitutionalismus. Dieser Frage soll im Folgenden am Beispiel der Verfassung der Republik der Vereinigten Staaten von Brasilien vom 24. Februar 1891 nachgegangen werden, dem Prototypus einer modernen lateinamerikanischen Verfassung mit US-Design, wie die offizielle Namensgebung verrät.

\section{Die Brasilianische Verfassung als US- Kopie}

Die textvergleichende Analyse bestätigt, was die Amtsbezeichnungen signalisieren: bei der Redaktion der »Constituição da República dos Estados Unidos do Brasil « von 1891 hat die »Constitution of the United States of America « von 1787 Pate gestanden. In diesem Urteil sind sich heute alle Verfassungsjuristen Brasiliens einig. Sie geben ohne weiteres zu, wenn auch mit dem Unterton des Bedauerns, dass seinerzeit die historischen Verfassungsväter von Rio de Janeiro das angloamerikanische Modell schlicht »kopiert« haben (Arinos, 1960:119; Leal, 1915:205).

Es gibt für dieses eingestandenermaßen eklektische Verhalten zumindest eine honorige Erklärung: man stand unter Zeitdruck. Nach Ausrufung der Republik am 15. November 1889 war schnelle Verfassungsgebung das Gebot der Stunde. Es galt, den republikanischen Systemwandel zu sanktionieren und den politischen Akteuren dieses Wandels die konstitutionelle Investitur zu sichern. In dieser bedrängten Situation sahen sich, wie noch zu zeigen sein wird, die republikanischen Verfassungsgeber gezwungen, auf eine konstitutionelle Neuschöpfung zu verzichten und sich an positiv Vorhandenem zu halten, also an bewährten in- wie ausländischen Vorbildern. So erklärt es sich, dass die Redaktoren der republikanischen Verfassung sich zunächst an der eigenen, nationalen Verfassungstradition orientiert haben, also vor allem an dem ersten republikanischen Verfassungswerk auf brasilianischem Boden, den Verfassungsentwurf der freien Republik von Rio Grande do Sul (»Projeto da Constituição da República Rio-Grandense«) vom 8. Februar 1843 (Russomano,1976:45) sowie, paradoxerweise, an der Verfassung des gerade gestürzten Kaiserreichs. Von eben dieser »Constituição Política do Império do Brasil« von 1824 übernommen wurden vor allem die stilistischen Elemente sowie alle national tragenden Normativen, wie z.B. das Staatsangehörigkeitsrecht.

In den machtpolitisch entscheidenden Fragen aber, also in der Frage der Organisation des neuen republikanischen Staatswesens und besonders der Frage der Gestaltung des Regierungsfrage, richtete sich der Blick auf das renommierte US-Modell. Von dieser Vorlage wurde das föderalistische Prinzip rezipiert - der Zentralstaat wurde aufgelöst, die ehemaligen »Provinzen« des Kaiserreichs wandelten sich in »Staaten« mit relativer Autonomie (Art.2 der »Constituição da República $\ll$ ) und verbanden sich unter dem Dach der »Union« zu »Vereinigten Staaten« (Art.1); ferner das Prinzip der drei Staatsgewalten - die Vierte, die moderierende Monarchengewalt (»Poder Moderador«) der Imperialverfassung wurde ersatzlos gestrichen; schließlich die zentrale präsidentialistische Regierungsform, also die uramerikanische Einrichtung eines Regierungssystems mit einem machtvollen Präsidenten an der Spitze der Exekutive 
(Art.44) und einer ausgeklügelten Verteilung der Zuständigkeiten und Machtbefugnisse zwischen Präsident und Kongress - wie in den USA sollte der Präsident regieren (Art.48) und der Kongress die Mittel bewilligen (Art.34); schließlich auch das Prinzip des Rechts- und Justizstaats, also die Etablierung einer starken unabhängigen Dritten Gewalt mit einem Supreme Court (»Supremo Tribunal Federal«) an der Spitze (Art.55,56). Dieser wurde wie in den USA mit der weitgehenden Kompetenz der Normenkontrolle ausgestattet.

Mit dem höchstrichterlichen Prüfungsrecht von Gesetzen und Regierungsakten wurden die angloamerikanische »rule of law «-Regel und das Prinzip des Supremats der Justiz implantiert, Institute also, die wie wahre Fremdkörper im überkommenen System der brasilianischen Gerichtswesens wirken mussten. Ferner wurden übernommen: das zweigliedrig aufgebaute Justizsystem, das »Justiça Federal « und »Justiça Estadual « organisch getrennt und die loyalen Gerichtsbarkeiten des Kaiserreichs, die »Juizes de Direito und »Juizes de Paz«, abgelöst hat (Mont’Alverne,1999:31); schließlich das System der Freiheiten der Person, womit die individuellen Grundrechte und Schutzansprüche endlich Verfassungsrang erhielten (Título IV). Erkenntlich sind auch viele Detailregelungen der US-Verfassung nachgemacht wie z.B. die 4-jährige Amtszeit des Präsidenten, allerdings ohne Wiederwahlmöglichkeit (Art.43); das Institut der Präsidentenanklage (»impeachment«) oder Amtsenthebung wegen Verantwortungsstraftaten (»crimes de responsabilidade«); die Ernennung der obersten Richter (»ministros $\ll$ ) durch den Präsidenten unter Zustimmung des Senats (Art.48 No.12) sowie deren Lebenszeitstellung (»vitalicidade«, Art.57); die Stellung des Präsidenten als Oberbefehlshaber der Streitkräfte (Art.48); das Zweikammerparlament, das zu Kaisers Zeiten »Assembléia Geral« hieß, wurde amerikanisch als »Congresso Nacional « organisiert; auch das Interventionsrecht der Union in Angelegenheiten der Länderstaaten wurde kopiert (Art.6) ebenso wie die Regelung der Hauptstadtfrage: nach amerikanischem Vorbild sollte die Hauptstadt der Republik neu errichtet werden, und zwar mit eigenem Bundesdistrikt (»Distrito Federal«) auf dem Planalto in Brasiliens geographischem Zentrum (Art.3). Noch weitere Parallelen lassen sich aufzählen. Doch schon dieser einfache semantische Verfassungsvergleich lässt Zweifeln an der Herkunft des brasilianischen Grundgesetzes aus Text und Geist der US-Verfassung wenig Raum. Ihrer Gesamtkonstruktion nach, so der semantische Anscheinsbeweis, stellt die Gründungsverfassung der brasilianischen Republik kein brasilianisches Original dar, sondern tatsächlich eine »Kopie«. Nur allzu deutlich lassen ihre Texturen die nordamerikanische Handschrift erkennen.

\section{Kontext und Determinanten des Verfassungstransfers}

Damit stellt sich noch einmal verstärkt die Frage nach einer Erklärung für diesen, aus nationalpolitischer wie kultureller Sicht hoch problematischen Verfassungstransfer, der sofort patriotische Kritik auf sich gezogen und als Akt unwürdiger »Servilität« und »Verrat an der Latinität« gebrandmarkt worden ist (Bonavides,1991:211). Offiziell aber hieß es, dass die notablen Verfassungsgeber der Republik, als sie seinerzeit in Rio de Janeiro scheinbar vorbehaltlos und ehrvergessen das US-Modell kopierten, aus 
staatsphilosophischer Einsicht gehandelt hätten, also aus hehrer republikanischer Staatsraison. Oder in Vollzug der liberaldemokratischen Aufklärung der Moderne. Oder zur Wahrung der wohlverstandenen Wirtschaftsinteressen der Nation. Inwieweit derartiger Legitimationsrhetorik Bedeutung zukommt, mag dahin gestellt sein. Überzeugender ist, nach konkreteren Erklärungen und Gründen für den Eklektizismus der republikanischen Verfassungsgeber zu suchen. Hier kommen vor allem auf zwei Gesichtspunkte in Frage, die am ehesten die verfassungsjuristische Einbürgerung des angloamerikanischen Verfassungsmodells plausibel machen. Gemeint ist einmal die Rolle des Zeit- und Erfolgsdrucks, dem das gesamte Verfassungsgebungsverfahren seit Ausrufung der Republik ausgesetzt gewesen ist, zum andern die Rolle der am Verfassungsgebungsverfahren maßgeblich beteiligten Juristen. Alles sieht danach aus, dass es diese Juristen gewesen sind, die letztlich den Ausschlag für die Übernahme des US-Modells gegeben haben.

Die äußere Erklärung für den unmittelbaren Modelltransfer aus den USA ergibt sich, wie gesagt, aus dem durch den Sturz der Monarchie entstandenen konstitutionellen Vakuum und dem dadurch bedingten Sachzwang zur Legitimierung des Machtwechsels und zur Investitur der republikanischen Machthaber. Die Verfassungsgebung stand in dieser Übergangsphase unter Erfolgsdruck. Die putschistische Machtergreifung (»golpe republicano«) hatte bekanntlich ohne Beteiligung des Volkes stattgefunden. Die von Marechal Manoel Deodoro da Fonseca angeführte Regierung war selbsternannt, also nur provisorisch an der Macht. Sie war gezwungen, in kürzester Zeit das revolutionäre Interregnum zu beenden und institutionelle Stabilität herzustellen. Diese politische Zwangslage spiegelt sich deutlich in der rasanten Abfolge der einzelnen institutionellen Akte wieder, die in nur 15 Monaten zur förmlichen Konstitutionalisierung der Republik geführt haben: Am 15. November 1889 erging das Dekret No.1, mit dem die Föderative Republik »als Regierungsform der brasilianischen Nation verkündet « wurde (Art.l). Art.2 verpflichtete die ehemaligen Provinzen, sich als »Vereinigte Staaten von Brasilien« zu konstituieren. Art.3 verlangte von den föderierten Staaten, sich »in Ausübung ihrer legitimen Souveränität« eine eigene Landesverfassung zu geben.

Mit Dekret Nr. 7 vom November 1889 wurden die Provinzialparlamente (Assembléias Provinciais) aufgelöst, Governeure eingesetzt, den neuen Gliedstaaten Autonomie eingeräumt. Weitere Dekrete führten das Wahlrecht ein und die Bundesjustiz. Dekret Nr. 29 vom 3. Dezember 1889 berief die Sonderkommission von Verfassungsjuristen zur Ausarbeitung eines Verfassungsvorentwurfs (»anteprojeto《), die sog. Fünferkommission (»Comissão dos Cinco«). Am 21. Dezember wurden deren Mitglieder ernannt und beauftragt, »schnellstmöglichst die endgültige Organisation der Vereinigten Staaten von Brasilien zu fixieren«. Im Januar 1890 trat sie erstmals unter Vorsitz von Saldanha Marinho zusammen. Fünf Monate darauf, am 24. Mai 1890, überreichte sie ihren Entwurf (»projeto primitivo«). Nach Überarbeitung des Entwurfs durch Rui Barbosa und Änderung des revidierten Entwurfs durch das Kabinett wurde dieser am 22. Juni 1890 (also 1 Monat später) durch Dekret Nr. 510 als eigener Entwurf der Provisorischen Regierung (»Substitutivo do Governo Provisorio «) veröffentlicht, später durch Dekret Nr. 914 vom 23.Oktober noch einmal ergänzt. 
Am 15. November 1890, also auf den Tag genau ein Jahr nach der Proklamation der Republik durch die Putschisten, trat der Kongress mit 205 Abgeordneten und 63 Senatoren erstmals zusammen. Das Parlament erhielt von der Provisorischen Regierung eine Frist von 20-30 Tagen, innerhalb derer die Verfassung zu verabschieden war. Am 22. November 1890 setzte der Kongress seine eigene Verfassungskommission ein (»Comissão dos 21 ), in der alle 21 Bundesstaaten mit Sitz und Stimme vertreten waren, und die fristgerecht drei Monate später, am 21. Februar 1891, nach Beratungen im Plenum, die Schlussfassung fertig stellte. Am 24. Februar 1891 erfolgte dann die feierliche Verkündung der Verfassung der Republik der Vereinigten Staaten von Brasilien in der Quinta da Boa Vista zu Rio de Janeiro. Der Abgeordnete und spätere Staatspräsident Prudente de Morais stellte fest: »Nach 15 Monaten tritt die Revolutionsregierung in den Zustand der Legalität. Unser Vaterland hat von nun an eine freiheitlich demokratische Verfassung, die es ihm ermöglicht, sich fortschrittlich zu entwickeln, Wohlstand zu erwerben und es in Südamerika dem nordamerikanischen Vorbild gleichzutun«(apud Bonavides, 1991: 225).

Die Verfassungsväter von Rio hatten damit schnelle und ganze Arbeit geleistet. Sie hatten sich pragmatisch, unter dem Druck der politischen Zeitenwende, für das seinerzeit modernste und fortschrittlichste Verfassungsmodell der Welt entschieden. Sie hatten $\mathrm{zu}$ diesem Zweck nur nachmachen müssen, was die Founding Fathers von Philadelphia höchst erfolgreich vorgemacht hatten. Dabei durften sie in der tiefen Überzeugung gehandelt haben, sich für eine Verfassung und mit ihr für ein politisches System entschieden zu haben, welches das normative Potential besaß, die Kräfte von Ordnung und Fortschritt im Lande zu bündeln und Brasilien in eine prosperierende Zukunft zu führen.

Es ist in diesem Zusammenhang kein Geheimnis, dass auch wirtschaftspolitische Gründe die Rezeption des US-amerikanischen Verfassungsrechts befördert haben. Schließlich war nicht zu verkennen, dass das liberale Verfassungsmodell die Grundlage für gemeinsame Wirtschaftsbeziehungen mit den USA gebildet hat, also als förderlich für die gegenseitigen Wirtschaftsinteressen anzusehen war. Wirtschaftshistorische Analysen (Moniz Bandeira,1997) haben überzeugend dargelegt, wie sehr die USA bereits gegen Ende des 19.Jahrhunderts mit ihrem industriekapitalistischen Wirtschaftsmodell in Brasilien eingedrungen waren, wie sehr sie seinerzeit zum Wohle ihrer eigenen Interessen die republikanische Bewegung in Brasilien wirtschaftlich wie diplomatisch gefördert und später, nach Ausrufung der Republik, sogar mit militärischen Mitteln alle Oppositionsbewegungen und die Obstruktionsversuche der monarchistischen Restauration zu verhindern gewusst haben. Ohne jeden Zweifel hat das vorhandene Beziehungsgeflecht mit den USA einen bestimmenden Einfluss auf das politische Brasilien und seinen republikanischen Wandel ausgeübt.

Damit nicht genug. Es sind keineswegs nur die in Politik und Constituinte vorherrschenden Opportunitätsstrategien und Verfahrenszwänge gewesen, die den Verfassungstransfer aus den USA erklärlich machen. Auch bestimmte Personenkreise im Aktionsradius der Macht und ihre staatstheoretischen Überzeugungen haben eine Rolle gespielt und am Ende sogar den Ausschlag gegeben. Gemeint sind die fachgelehrten Juristen, die die Verfassungsgebung beraten haben, also hauptsächlich die 
illustren Mitglieder der genannten Fünferkommission Saldanha Marinho, Américo Brasiliense, Santos Werneck, Rangel Pestana und Pereira de Magalhães sowie vor allem der überragende Rui Barbosa.

Diese personalistische Sicht der Dinge wird allerdings erst einleuchtend, wenn man sich den Ausnahmestatus und die herausragende Rolle der »juristas « in der Verfassungsgebenden Versammlung von 1890/91 vor Augen hält. Es ist kein Geheimnis, dass nur sehr wenige Mitglieder der Constituinte über die juristischen Kompetenz verfügt haben, ihre gesetzgeberische Aufgabe zu erfüllen, also auf professionellen juristischen Beistand angewiesen waren. Dieses Dilemma erklärt sich aus der juristischen Zeitgeschichte nicht nur Brasiliens, sondern ganz Lateinamerikas. Es ist ersichtlich die Folge des juristischen Bildungsnotstandes gewesen, mit dem seit ihrer Unabhängigkeit alle Staaten Lateinamerikas zu kämpfen hatten, als sie vor der Aufgabe standen, sich nationale Gesetze und Verfassungen zu geben. Überall fehlten eigene Vorbilder und Rechtstraditionen, die in der revolutionären Situation hätten Orientierung sein können, und entsprechend mangelte es an fachlich gebildeten Juristen, die den Beruf der Zeit für Gesetzgebung und Rechtsreform hätte ausüben können. Die vorhandenen Universitäten Lateinamerikas, die immerhin als Rechtsfakultäten gegründet worden waren (Steger,1989:423), hatten sich als unfähig erwiesen, modernes Recht zu vermitteln. Das galt auch für die ersten Universitäten Brasiliens, die im Jahre 1827 als Faculdades de Direito in Olinda und São Paulo gegründet worden waren, vornehmlich aber als traditionalistische Außenstellen Coimbras und nicht als Vermittler liberaler Rechtstraditionen fungierten (Venancio Filho,1982:13). So ist es zu verstehen, dass in der Gründerzeit der lateinamerikanischen Nationalstaaten nur wenige universal gebildete Juristenpersönlichkeiten zur Verfügung gestanden haben, die den Anforderungen der Gesetzgebungsaufgabe gewachsen waren wie Juan Bautista Alberdi und Vélez. Sársfield in Argentinien, Andrés Bello in Chile, Ignacio Vallarta in Mexiko und eben das brasilianische Dreigestirn Teixeira de Freitas, Clóvis Bevilaqua und Rui Barbosa. Sie sind es gewesen, die in der Rolle von Vermittlern moderner europäischer Rechtskultur zu den eigentlichen Wegbereitern und Schöpfern der großen nationalen Gesetzgebungswerke Lateinamerikas geworden sind (Paul,1995).

Angesichts dieses Hintergrundes wird die überragende Rolle Rui Barbosas als Kronjurist der Interimsregierung und universaljuristisch gelehrter Kopf in den diversen Gremien der Verfassungsgebung am ehesten verständlich. Der gebürtige Bahianer Rui Barbosa gilt heute, wenngleich nicht unbestritten (Bonavides 1991:217), als der geistige Urheber und Redaktor der republikanischen Verfassung und vieler Dekrete der Provisorischen Regierung (Lacombe,1949). Er diente als Jurist zunächst dem Kaiserreich, dann der Republik als Minister der Provisorischen Regierung, später als Berater und Anwalt. Er hat sich häufig als Bewunderer der angelsächsischen Rechtskultur bekannt, hatte Franklin, Adams, Jefferson, Hamilton und Madison studiert (Smith,1945), war fasziniert von der Rechtsprechung des Supreme Court, vor allem von der »florecência prodigiosa da criação da Carta Federal dos Estados Unidos da América do Norte« (»der herrlich aufblühenden Schöpfung der Bundesverfassung der Vereinigten Staaten von Nordamerika«), wie er in seinen berühmten Comentários à Constituição schrieb. Er hat sich innerhalb der Regierung und der beschließenden Gre- 
mien nachhaltig für die Gestaltung der republikanischen Verfassung nach angloamerikanischem Vorbild eingesetzt. In seinem Hause und unter seiner Regie tagte regelmäßig die erwähnte »Fünferkommission«, die den Vorentwurf erarbeitete. Er war es, der den Vorentwurf umschrieb (Facsímile,1890), dann eigenhändig die Redaktion des Regierungsentwurfs besorgte und diesen in der Constituinte verteidigte, wo er à brasileira »o azul dos novos horizontes, o oxygénio poderoso da civilização americana«( »das Blau neuer Horizonte, den mächtigen Sauerstoff der amerikanischen Zivilisation «) pries. Auch wenn er immer wieder in Rücksicht auf die nationale Gefühlslage seine angloamerikanistische Präferenz relativiert hat, muss in ihm der gelehrte Jurist gesehen werden, der durch die Überlegenheit seines juristischen Sachverstands und die Unwiderstehlichkeit seiner Rhetorik dominanten Einfluss auf die Entscheidungen der Verfassungsgebung genommen hat.

\section{Schein und Sein des brasilianischen Konstitutionalismus}

Verfassungen sind bekanntlich mehr als bloß formale, rechtliche Rahmenordnungen eines Staates. Sie sind auf ihre juristisch verschlüsselte Weise immer auch Ausdruck der inneren Verfasstheit einer Nation, also immer auch Ausdruck dessen, was »nationale Identität « genannt wird - eine emotionale Größe, die im patriotischen Seelenhaushalt zumal der jungen Nationen Lateinamerikas fest verankert ist. So gesehen, muss es nicht verwundern, dass in den Gründerjahren der brasilianischen Republik die »servile Nachahmung « der US-Verfassung erhebliche emotionale Widerstände geweckt hat und als Verrat an Latinität und Brasilianität (»brasilianidade«) empfunden worden ist. Konsequent formierte sich der nationalpatriotische Widerstand als staatstheoretische Opposition im Verfassungsgebungsprozess. Unter Berufung auf die nationalen Traditionen des positivistischen Konstitutionalismus forderte die nationalpatriotische Fraktion an Stelle der »demokratischen Republik « nach angloamerikanischem Vorbild die »diktatoriale Republik« im Sinne von Auguste Comte und des Gaúcho-Republikanismus von Rio Grande do Sul (Hentschke, 2003). Wie sich dann zeigte, sind die Nationalpatrioten in den Schlussabstimmungen der Constituinte unterlegen gewesen. Sie haben dort die Verabschiedung des von ihnen bekämpften liberalen Verfassungswerks nicht verhindern können. Doch sind sie keineswegs leer ausgegangen: es ist ihnen gelungen, ihrer positivistischen Position ebenfalls offizielle Geltung zu verschaffen, zwar nicht im Text der Verfassung selbst, dafür aber höchst spektakulär in der symbolischen Außendarstellung der Republik. In Form eines Handstreichs haben sie sich des zentralen Staatssymbols und Herzstïcks der Republik, nämlich ihrer Fahne, der bandeira nacional, bemächtigen können.

Wie es dazu kommen konnte, ist denkwürdig genug, wie ein kurzer Blick auf die hier gemeinten Ereignisse in den Tagen vom 15. bis 24. November des Jahres 1889 belegt. Zu erinnern ist daran, dass tatsächlich das, was später »republikanische Revolution « genannt werden sollte, zunächst auf den Straßen der Hauptstadt in Gestalt eines spontan entbrannten Streits um das Aussehen der republikanischen Fahne stattgefunden hat. Es ging um das heraldische Design des republikanischen Staatssymbols, also erkenntlich um eine nationale Herzensangelegenheit, der umso größere Bedeu- 
tung zukam, als klar wurde, dass die »Revolution « gewaltlos und nur symbolisch stattfand, also ohne Barrikaden, Heldentaten und tote Helden, lediglich in Gestalt von paradierenden Soldaten und aufgeregt sich versammelnden Bürgern (Gilberto Freyre,1974). In diesen bewegten Tagen des »Krieges der Hurra-Rufe« (»guerra dos vivas «, Murilo de Carvalho,1990:36) wurde ein Fahnentuch durch die Straßen getragen, welches eine schockierende Ähnlichkeit mit dem US-Banner »stars and stripes « aufwies und sich als Fahne des liberalen Republikanerklubs "Lopes Trovão" herausstellte. Als dann eben diese Fahne auch noch am Mast des Parlamentsgebäudes der Hauptstadt aufgezogen wurde, war für die Nationalpatrioten der casus belli gegeben. Deren positivistische Avantgarde, bestehend aus Oberstleutnant Benjamin Constant de Magalhães, dem Lehrer des Positivismus an der Militärakademie »Escola Superior de Guerra «u Rio de Janeiro und Chefideologen in der Provisorischen Regierung des Marschalls Deodoro da Fonseca, sowie aus den beiden Direktoren des Apostolats der Positivistischen Kirche, Teixeira Mendez und Miguel Lemos, brandmarkte die Klubfahne der Liberalen als hinterhältigen Vaterlandsverrat.

Über Nacht gestaltete weisungsgemäß der Maler Décio Villares, nach Vorbild der »Politischen Fahne des Okzidents«(»Drapeau Politique de l'Occident«, Auguste Comte, 1967:I,387;IV,422) und unter Verwendung der heraldischen Insignien der lusobrasilianischen Tradition (Olavo Coimbra,2000), das bekannte astronomische Design mit der positivistischen Legende »Ordem e Progresso«, und eiligst, schon 4 Tage nach der Proklamation, am 24. November 1889, wurde dieser Entwurf per Gesetzesdekret im Diário Oficial als Staatsflagge der Ersten Brasilianischen Republik verkündet. Damit war auf legale Weise die programmatische »Ordnung und Fortschritt «- Devise zum Taufspruch der Republik geworden (Paul,1997). Alle nach 1891 unternommenen Versuche der Liberalen, das offensichtlich verfassungswidrige Fahnengesetz zu annullieren, sind an beharrlichen Widerständen im Kongress gescheitert. Noch heute verkündet, wo immer sie weht, die ,schönste Fahne der Welt' das positivistische Glaubensbekenntnis, das nach Comtescher Vorstellung unverkennbar dem Staatsideal der »diktatorialen Republik « huldigt.

Was hier als Kuriosität und Anekdote aus den Gründerjahren der Republik erscheint, sagt in Wahrheit Grundlegendes über die Eigentümlichkeit des brasilianischen Konstitutionalismus aus. Mit der Fahne war nach herrschender Auffassung das positivistische Staatsideal zum offiziellen Staatsziel erhoben worden und hatte Verfassungsrang erlangt. Damit war zugleich die »diktatoriale « Staatsraison der Positivisten in konstitutionelle Realkonkurrenz zur demokratischen Staatsraison der Liberalen getreten. Unversehens hatte der Fahnenstreit die staatstheoretische Dichotomie zum Vorschein gebracht, die das brasilianische Verfassungswesen in charakteristischer Weise prägen und schließlich Verfassungsgeschichte machen sollte. Vor die Wahl gestellt, hatte das politische Brasilien die konstitutionelle Grundentscheidung zu treffen, welches der beiden konkurrierenden Republikkonzepte für die politische Gestaltung des Landes maßgeblich sein sollte. In der Sache ging es um die höchst praktische Frage, welche Regierungsform am ehesten den politischen Realitäten des Landes entsprechen und die Dynamik des politischen Machtprozesses bestimmen sollte. 
Der diagnostische Rückblick auf die Geschichte der Republik ergibt, dass das politische Brasilien dieser Grundentscheidung aus dem Weg gegangen ist und das Heil in einem Scheinkompromiss gesucht hat. Dieser hat darin bestanden, dass die wechselnden Regierungsregime, ungeachtet ihrer ideologischen Couleur, die demokratische Regierungsform immerfort de iure deklariert, de facto hingegen die caudillistische Tradition praktiziert haben. In der Geschichte der brasilianischen Republik ist es den militärischen wie zivilen Staatspräsidenten regelmäßig gelungen, ihre rechtliche Vorrangstellung in faktische Vormachtstellung zu verwandeln. Sie alle haben es als vorteilhaft gefunden, ihre »unverfälscht autoritäre Regierung hinter einer pseudokonstitutionellen Fassade zu verbergen « (Löwenstein,1975:442). Mit anderen Worten: nicht die geschriebenen Staatsverfassungen haben in Brasilien die Dynamik der politischen Machtprozesse geprägt, sondern die ungeschriebenen Verhaltensgesetze der Macht und der regierenden Regime, also die sog. »Parallelverfassungen«, die als die realen Verfassungen des Landes gelten.

Am Anfang dieser Realgeschichte des brasilianischen Konstitutionalismus hat die idealistische Willkür der republikanischen Verfassungsväter von 1891 gestanden, das liberal egalitäre Verfassungsmodell der USA der auf Ungleichheit basierenden Gesellschaft Brasiliens zu implantieren. Damit hat die brasilianische Entwicklung nur das bestätigt, was in ganz Lateinamerika verwurzelte öffentliche Überzeugung ist, nämlich »dass keine angemessenen Konzeptionen für die Anwendung oder Durchsetzung des Staatsmodells der sog. Okzidentalen Welt auf eine andere historische Realität existieren $\ll($ Reyes Alvarez,2003:429).

\section{Ausblick}

Die Frage ist, ob heute, ganze 112 Jahre danach, reale Chancen bestehen, den diskreditierten Neo-Präsidentialismus zugunsten der konstitutionellen Regierungsform abzulösen. Die Aussichten auf eine derartige Verfassungsrevolution sind denkbar schlecht. Die bisher vom Kongress verabschiedeten 38 Änderungsnovellen, die sämtlich als Reformen der kühnen Verfassung von 1988 deklariert worden sind, haben deutlich Anderes und häufig Gegenteiliges im Sinn gehabt (Paul,1995). Ohnehin hätten sich, so die Lektion aus der Verfassungsgeschichte der Republik, die Hoffnungen der Nation vor allem auf die informelle, die »parallele« Verfassung zu richten, von der nach aller Erfahrung die Kraft wirklicher Veränderung auszugehen hätte. Deren Texturen aber sind nach wie vor Verschlusssache der »herrschenden Klasse Brasiliens « (Darcy Ribeiro,1980:13), also dem öffentlichen Diskurs entzogen. Damit ist am Ende die quälende Frage aller konstitutionellen Fragen aufgeworfen, warum es den Brasilianern bis heute nicht gelungen ist, ihr Verfassungsproblem zu lösen (Miguel Reale, 1985). Die Antwort kennen bislang nur die politischen Märchenerzähler. Sie liegt in der suggestiven Bildersprache verborgen, welche die großen Literaten Lateinamerikas verwenden, um magische Wirklichkeiten zu beschwören. Noch keinem Neugierigen, so besagt es die treffliche Metapher eines intimen Kenners der Verhältnisse (Revueltas,1975:70), ist es vergönnt gewesen, »die schwarze Katze der Verfassung im dunklen Zimmer der Politik« ausfindig zu machen. 


\section{Literatur:}

Arinos de Melo Franco (1960), Curso de Direito Constitucional, Rio de Janeiro

Barbosa, Rui (1890), Projeto da Constituição de 1891. Facsímile, in: Rui Barbosa e a Constituição de 1891, Fundação Casa de Rui Barbosa, Rio de Janeiro 1985

Barbosa, Rui (1932), Comentários à Constituição Federal Brasileira, São Paulo, vol. 1

Bonavides, Paulo - Paes de Andrade (1991), História Constitucional do Brasil, São Paulo

Comte, Auguste (1967), Système de Politique Positive ou Traité de Sociologie, instituant la Religion de l'Humanité. Réimpression de l'Édition 1851-81, Tome Premier et Quatrième

Dippel, Horst (Hrsg.), Verfassungen der Welt, 5 vols. , München o.J.

Freyre, Gilberto (1974), Ordem e Progresso. Rio de Janeiro, 3a. ed.

Grimm, Dieter ( 1991), Die Zukunft der Verfassung, Frankfurt

Hentschke, Jens (2003), Positivism gaúcho-style: Júlio de Castilhos' Dictatorship and its impact on State \& Nation- Building in Vargas' Brazil, Newcastle

Lacombe, Américo Jacobina (1949), Rui Barbosa e a Primeira Constituição da República, Rio de Janeiro: Casa de Rui Barbosa

Leal, Aurelino (1915), História Constitucional do Brasil, Rio de Janeiro

Löwenstein, Karl ( 1975), Verfassungslehre, Tübingen, 3.Aufl.

Mont'Alverne, Martonio (1999), Staat und Justiz in Brasilien. Schriften der Deutsch-Brasilianischen Juristenvereinigung Bd.28, Frankfurt

Moniz Bandeira, Luiz Alberto (1997), Relações Brasil-EUA no Contexto da Globalizão, Vol. I, Presênça dos EUA no Brasil, ed.revista

Murilo de Carvalho, José (1990), A Formação das Almas. O Imaginário da República no Brasil, São Paulo

Olavo Coimbra, Raimundo (2000), A Bandeira do Brasil, Rio de Janeiro, 3a.ed.

Paul, Wolf (1994), Verfassungsgebung und Verfassung, in: Brasilien heute. Dietrich Briesemeister u.a.(Hrsg.), Bibliotheca Ibero-Americana Bd.53, Frankfurt

Paul, Wolf (1995), Kurzbiographien von Alberdi, Barbosa, Bello, Bevilaqua, Teixeira de Freitas, Vélez Sársfield, u.a. in: Juristen. Ein biographisches Lexikon. Michael Stolleis (Hrsg.), München

Paul, Wolf (Hrsg. 1995), Verfassungsreform in Brasilien und Deutschland. Schriften der Deutsch-Brasilianischen Juristenvereinigung Bd. 24, Frankfurt

Paul, Wolf (1997), Ordem e Progresso. Entstehung und Deutung des brasilianischen Fahnensymbols, in: Freundesgabe für Friedrich Kübler, hrsg. von Heinz-Dieter Assmann u.a., Heidelberg; auch: Ordem e Progresso. Origem e Significado dos Símbolos da Bandeira Nacional Brasileira. Homenagem aos 500 Anos do Brasil, in: Revista do Tribunal Regional do Trabalho da Oitava Região No.65, Jul./Dez. 2000, Belém (Pará), págs. 161 - 175

Reale, Miguel (1985), Por uma Constituição Brasileira, São Paulo

Revueltas, José (1997), Die schwarze Katze der Verfassung im dunklen Zimmer der mexikanischen Politik, Frankfurt

Reyes Alvarez, Jaime (2003), Ars Regnandi. Regierungsstabilität und Herrschaftskrisen in Iberoamerika. Am Beispiel von Argentinien und Chile. Diss. Frankfurt am Main

Ribeiro, Darcy (1979), Über das Selbstverständliche, in: Darcy Ribeiro, Unterentwicklung, Kultur und Zivilisation, Frankfurt

Smith, Carleton Sprague (1945), Os Livros Norte-Americanos no Pensamento de Rui Barbosa, Rio de Janeiro

Steger, Hanns-Albert (1989), Zur Kultursoziologie der westeuropäischen und lateinamerikanischen Universitätssysteme im 19. und 20. Jahrhundert, in: Hanns- Albert Steger, Weltzivilisation \& Regionalkultur, München

Russomano, Victor (1976), História Constitucional do Rio Grande do Sul, Porto Alegre

Venancio Filho, Alberto (1982), Das Arcadas ao Bacharelismo, São Paulo 